\title{
Quasiperiodic criticality and spin-triplet superconductivity in superconductor-antiferromagnet moiré patterns
}

\author{
Maryam Khosravian $\odot$ and J. L. Lado $\odot$ \\ Department of Applied Physics, Aalto University, 00076 Aalto, Espoo, Finland
}

(Received 4 November 2020; revised 21 January 2021; accepted 3 March 2021; published 19 March 2021)

\begin{abstract}
Quasiperiodicity has long been known to be a potential platform to explore exotic phenomena, realizing an intricate middle point between ordered solids and disordered matter. In particular, quasiperiodic structures are promising playgrounds to engineer critical wave functions, a powerful starting point to engineer exotic correlated states. Here we show that systems hosting a quasiperiodic modulation of antiferromagnetism and spin-singlet superconductivity, as realized by atomic chains in twisted van der Waals materials, host a localization-delocalization transition as a function of the coupling strength. Associated with this transition, we demonstrate the emergence of a robust quasiperiodic critical point for arbitrary incommensurate potentials, which appears for generic relative weights of the spin-singlet superconductivity and antiferromagnetism. We show that inclusion of residual electronic interactions leads to an emergent spin-triplet superconducting state, which gets dramatically enhanced at the vicinity of the quasiperiodic critical point. Our results put forward quasiperiodicity as a powerful knob to engineer robust superconducting states, providing an alternative pathway towards artificially designed unconventional superconductors.
\end{abstract}

DOI: 10.1103/PhysRevResearch.3.013262

\section{INTRODUCTION}

Unconventional superconductivity [1,2] encompasses one of the most exotic states found in quantum materials. In particular, recent interest in topological superconductors hosting Majorana states has been boosted by their potential for topological quantum computing [3,4]. However, unconventional superconductivity and, in particular, spin-triplet superconductivity remains a highly elusive state in natural compounds [5-9], with a few exceptions such as doped $\mathrm{Bi}_{2} \mathrm{Se}_{3}$ [10-13], $\mathrm{UTe}_{2}$ [14], and $\mathrm{UPt}_{3}$ [15]. Whereas several compounds have been identified as a potential candidate for spin-triplet superconductivity [16-21], finding generic mechanisms for its engineering still remains a challenge. To date, a highly successful strategy for engineering spin-triplet superconductors consists of focusing on materials with potential coexisting magnetic and superconducting orders [22,23]. This procedure has been heavily exploited for the engineering of Majorana bound states with a variety of platforms [24-31]. Most of these schemes have relied on engineering periodic structures with competing orders, while its study in nonperiodic systems has remained relatively unexplored [32,33]. In stark contrast, the study of conventional superconductivity in disordered [34-36] and quasiperiodic [37-40] systems has a long history, especially in demonstrating the potential critical advantages of nonperiodicity for engineering robust superconducting states.

Published by the American Physical Society under the terms of the Creative Commons Attribution 4.0 International license. Further distribution of this work must maintain attribution to the author(s) and the published article's title, journal citation, and DOI.
Quasiperiodic patterns display a never-repeating arrangement of elements $[41,42]$, yet they host a long-range order. Due to the lack of conventional periodicity, standard band structure arguments no longer hold, and their electronic structure exhibits a notably rich behavior [43], such as the presence of confined states [44-46], fractal dimensions [47-49], pseudogap in the density of states [50-53], and unconventional conduction properties [54-57]. More importantly, the incommensurate structure of quasicrystals has prominent effects on the electron eigenstates. Incommensurate potentials give rise to electronic wave functions that are extended, localized, or critical [58-61], and ultimately can host topological states of matter fully associated to the quasiperiodicity $[62,63]$.

Here we demonstrate that quasiperiodic patterns arising from a combination of spin-singlet superconductivity and antiferromagnetism provide a powerful platform to engineer spin-triplet superconductivity. In particular, we show that this antiferromagnetic-superconductor pattern hosts a localization-delocalization phase transition, with an associated quasiperiodic critical point with multifractal wave functions. We further show that upon inclusion of residual interactions, a spin-triplet superconducting state emerges that gets dramatically enhanced at the proximity of the localization-delocalization critical point. Our results show that magnetic-superconducting quasiperiodic patterns, as those found in atomic chains in twisted van der Waals materials, provide a different mechanism to engineer unconventional superconducting states by exploiting quasiperiodic criticality.

Our manuscript is organized as follows. Section II introduces a realization of our model, and we show the emergence of a critical point in quasiperiodic superconductorantiferromagnet patterns, separating the extended and 


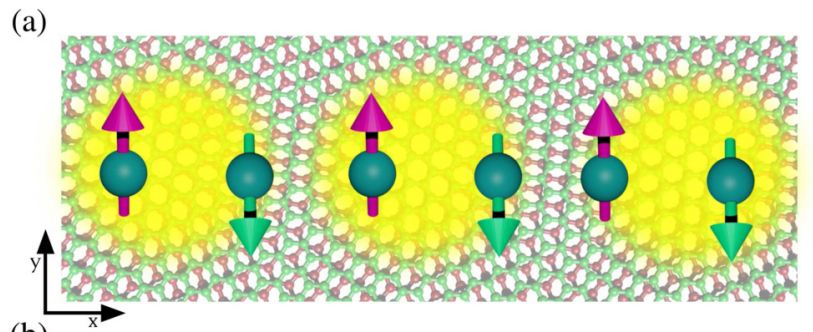

(b)

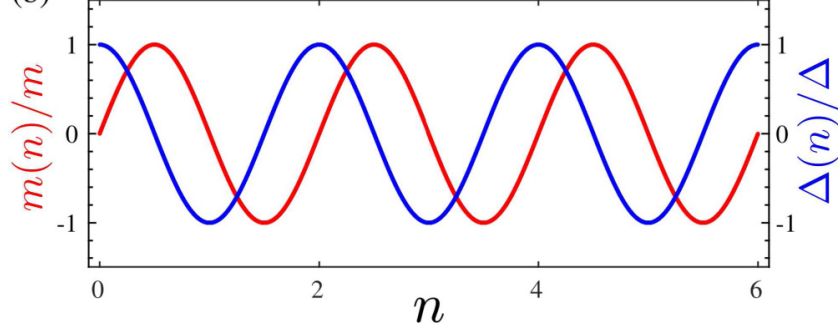

FIG. 1. (a) Engineered antiferromagnetic atomic chain on top of a superconducting twisted graphene bilayer. The twisted system shows a spatially modulated superconducting state (yellow), which coexists with the spatially modulated antiferromagnetism of the chain. (b) The modulation of the superconducting $\Delta(n)=$ $\Delta \cos (\Omega n)$ and antiferromagnetic $m(n)=m \sin (\Omega n)$ order parameters along the chain direction, associated to the Hamiltonian of Eq. (1).

localized regime. In Sec. III, we show that interactions give rise to a spin-triplet superconducting state, and we analyze its dependence with respect to the details of the quasiperiodic modulation. In Sec. IV, we demonstrate the robustness of the interaction-induced spin-triplet superconducting state with respect to perturbations in the quasiperiodic Hamiltonian. Finally, in Sec V, we summarize our conclusions.

\section{ANTIFERROMAGNET-SUPERCONDUCTOR QUASIPERIODIC CRITICALITY}

The system that we will study combines a spatially modulated antiferromagnetism and superconductivity, as shown in Figs. 1(a) and 1(b). This type of spatially modulated parameters appears in generic twisted two-dimensional materials that combine superconductivity and magnetism [31]. Here we will focus on a specific case in which the system is purely one dimensional. This situation can be realized by taking a twisted graphene multilayer in a superconducting state [64-70], whose superfluid density follows the modulation of the moiré pattern, and depositing an array of adatoms on top of it [71-78] [Fig. 1(a)]. The adatoms will have a longrange antiferromagnetic order stemming from the graphene Ruderman-Kittel-Kasuya-Yosida (RKKY) interaction [79], leading to a one-dimensional antiferromagnetic state [80-82]. Both electronic orders will have a modulation following the moiré pattern, effectively realizing a one-dimensional model with modulated antiferromagnetism and superconductivity [83-86].

We describe this system combining a modulated superconducting and antiferromagnetic exchange by the following effective Hamiltonian [87-90]:

$$
\begin{aligned}
\mathcal{H}= & t \sum_{n, s} c_{n, s}^{\dagger} c_{n+1, s}+\text { H.c. } \\
& +m \sum_{n, s, s^{\prime}} \sigma_{z}^{s, s^{\prime}}(-1)^{n} \sin (\Omega n) c_{n, s}^{\dagger} c_{n, s^{\prime}} \\
& +\Delta \sum_{n} \cos (\Omega n) c_{n, \uparrow}^{\dagger} c_{n, \downarrow}^{\dagger}+\text { H.c. }
\end{aligned}
$$

where $c_{i, s}^{\dagger}\left(c_{i, s}\right)$ denotes the fermionic creation (annihilation) operator for site $n$ and spin $s$, and $\sigma_{z}$ is the spin Pauli matrix. The first term denotes the kinetic energy of the system, the second term denotes the spatially modulated antiferromagnetism, and the third term corresponds to the modulated superconductivity. The parameters $\Delta$ and $m$ are responsible for the strength of the modulation corresponding to the antiferromagnetism and superconductivity, respectively, and $\Omega$ is the wavelength of the modulation. The model of Eq. (1) assumes that the proximity-induced superconducting state will be stronger when the magnetism is weaker [Fig. 1(b)], as often happens for spin-singlet superconductivity. In the following, the $s$-wave superconducting order will be taken as a parameter of the model as stemming from proximity. When interactions are included, a different superconducting order can appear on top of the proximitized one. This additional superconducting order arising when interactions are included will be called interaction-induced superconducting order. For convenience, we will parametrize the superconducting and antiferromagnetic strength as $m=\lambda \sin \alpha$ and $\Delta=\lambda \cos \alpha$, so that the net strength of the quasiperiodic modulation can be defined by the parameter $\lambda=\sqrt{m^{2}+\Delta^{2}}$.

For irrational values of $\Omega /(2 \pi)$, the model of Eq. (1) lacks translational symmetry and thus does not accept a description in terms of Bloch states. As a result, the eigenstates of this Hamiltonian are not guaranteed to be extended states, as the Hamiltonian is inherently nonperiodic. Whereas random disorder creates localization at arbitrarily small coupling constants in one dimension [91], quasiperiodic patterns are known to give rise to a localization transition at finite coupling constant $[59,92]$. In particular, it is worth noting that for $\Delta=0$, our model is mathematically equivalent to the AubryAndre-Harper (AAH) model [59]. Therefore, in the limit of $\Delta=0$, the previous model will have a localization transition at $m=2 t$, so that for $m<2 t$, all the states will be extended, and for $m>2 t$, all the states will be localized. As we show below, the generalized model of Eq. (1) with $\Delta \neq 0$ shares many of the characteristics of the AAH model, in particular a critical transition at the finite coupling constant.

We now address the localization-delocalization transition in the previous model. To determine the extended and localized nature of the states, we compute the inverse participation ratio (IPR) of each eigenstate $\Psi_{n}$ as

$$
\mathcal{P}_{n}=\sum_{i}\left|\Psi_{n}(i)\right|^{4},
$$

where $i$ runs over all the components of each eigenstate. For localized states whose wave function spans a certain number of sites $L$, for a system of size $N$, the value of the IPR is a finite nonzero number. In stark contrast, for extended states, 

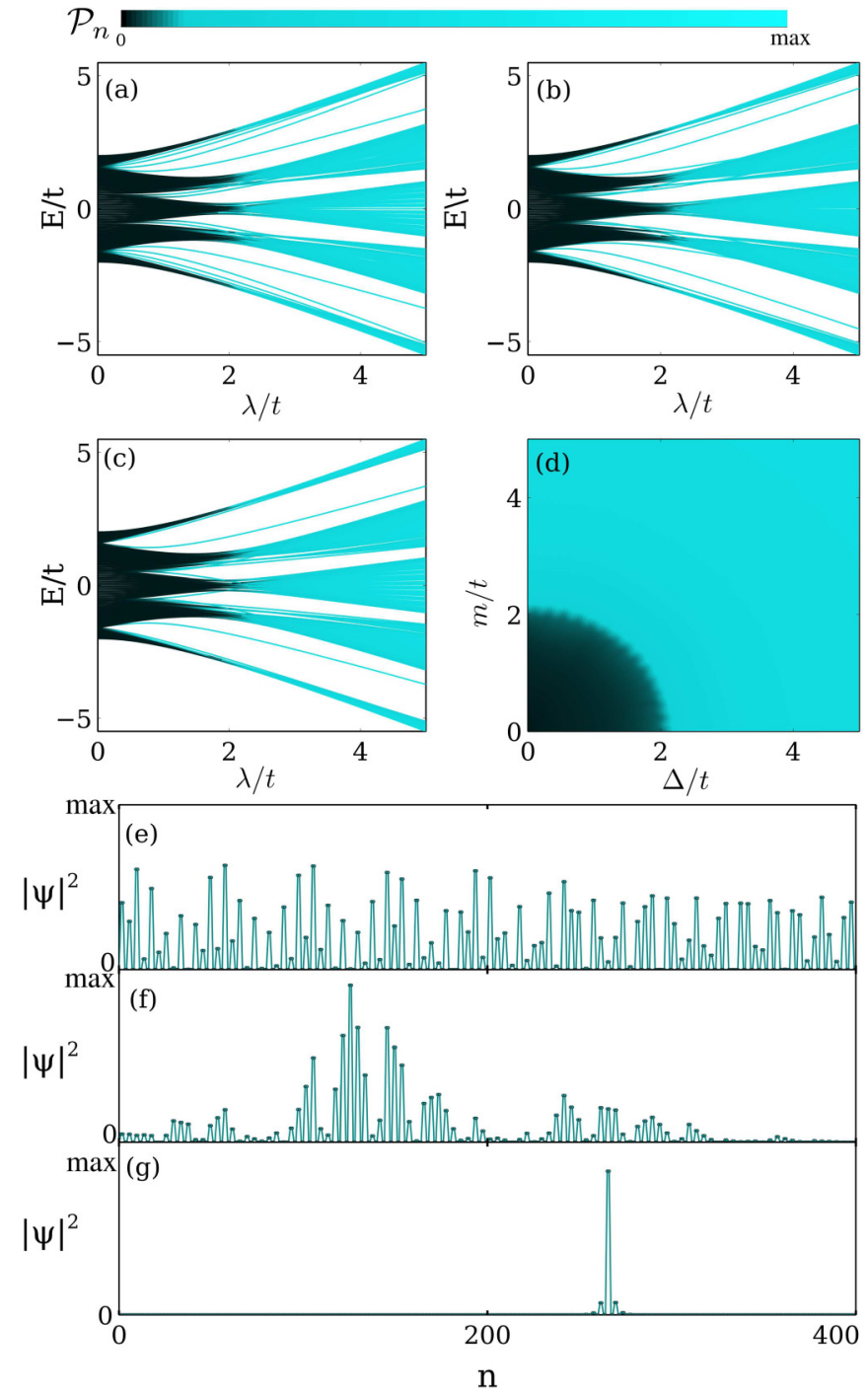

FIG. 2. (a)-(c) IPR for every state as a function of the modulation strength, with (a) $\alpha=\pi / 3$, (b) $\alpha=\pi / 4$, and (c) $\alpha=\pi / 6$. It is observed that the the localization transition takes place for $\lambda=2 t$ in all instances. (d) IPR averaged over all the states as a function of $m$ and $\Delta$, showing a phase boundary following $\Delta^{2}+m^{2}=4 t^{2}$. (e)-(g) The spatial distribution of the wave function in the (e) extended, (f) critical, and $(\mathrm{g})$ localized regimes.

the value of the IPR scales as $1 / N$ becomes zero in the thermodynamic limit.

Let us now explore the model of Eq. (1) and, in particular, analyze how the localization of the states evolves as we increase the strength of the quasiperiodic modulation $\lambda$. We show in Fig. 2(a) the evolution of the IPR for the different eigenstates, as a function of the modulation strength $\lambda$ for $\alpha=$ $\pi / 3$, which corresponds to taking $\Delta / m \approx 0.57$. In particular, as shown in Figs. 2(a)-2(c), all the states remain extended until $\lambda=2 t$ is reached, at which point all become localized. Note that the single in-gap modes that remain localized all the time correspond to topological edge states [62,63]. This can be systematically studied by looking at the average IPR of the states as a function of $\Delta$ and $m$. This is shown in Fig. 2(d), where it can be seen that a boundary with the functional form
$\Delta^{2}+m^{2}=\lambda^{2}=4 t^{2}$ separates the localized region from the extended region. The transition's independence with respect to $\alpha$ can be rationalized from a low-energy model. In particular, for a tight-binding chain with antiferromagnetism and superconductivity, the low-energy model consists of a fourcomponent Dirac equation [87-90,93]. The antiferromagnetic $m$ and superconducting $\Delta$ order parameters enter this lowenergy model as two inequivalent masses $m(x)$ and $\Delta(x)$ in the Dirac equation. Thus, from the low-energy perspective, the quasiperiodic model can be understood as a Dirac equation in which two mass terms $m(x)$ and $\Delta(x)$ are modulated in space. In particular, performing a local spinor rotation of the Dirac model, we reach an effective model with a single Dirac equation with a mass term $\chi(x)=\sqrt{m(x)^{2}+\Delta(x)^{2}}$. This spinor rotation does not change the localized or delocalized nature of the eigenstates. By definition of $m(x) \sim \lambda \cos \alpha$ and $m(x) \sim \lambda \sin \alpha, \chi(x)$ is independent of $\alpha$, and therefore the localization-delocalization nature becomes independent of $\alpha$.

At the previous phase boundary separating the extended from localized states, wave functions with critical behavior emerge. The different nature of the extended, localized, and critical states can be easily observed by plotting individual wave functions. In particular, we show in Figs. 2(e)-2(g) the wave function closest to charge neutrality for an extended [Fig. 2(e)], critical [Fig. 2(f)], and localized [Fig. 2(g)] regime. Whereas the extended wave functions span over the whole system [Fig. 2(e)], localized wave functions are strongly localized in a few lattice sites [Fig. 2(g)]. The critical wave function of Fig. 2(f) is characterized by multifractal revivals [94-100]. This will become especially important in the next section, as the multifractal behavior of the states will substantially increase the impact of interactions in the system.

\section{INTERACTION-DRIVEN SPIN-TRIPLET SUPERCONDUCTIVITY}

Let us now move on to consider the impact of interactions in the previous quasiperiodic system. In particular, we will show that the inclusion of interactions will lead to spintriplet superconductivity, where the criticality driven by the quasiperiodic pattern can be used as a knob to enhance the superconducting order parameter close to the critical point. Local interactions of the form $\mathcal{H}_{\text {int }} \sim \sum_{n} c_{n, \uparrow}^{\dagger} c_{n, \uparrow} c_{n, \downarrow}^{\dagger} c_{n, \downarrow}$ are already accounted for in the staggered antiferromagnet and superconducting terms of the Hamiltonian. Therefore, we will now consider the effect of residual nonlocal interactions, in particular nearest-neighbor density-density interactions. For that reason, we will now include an interaction term in our Hamiltonian of the form

$$
\mathcal{H}_{V}=-V \sum_{n}\left(\sum_{s} c_{n, s}^{\dagger} c_{n, s}\right)\left(\sum_{s^{\prime}} c_{n+1, s^{\prime}}^{\dagger} c_{n+1, s^{\prime}}\right),
$$

where $V$ controls the strength of the nearest-neighbor attractive interaction. In the following, we will solve the previous Hamiltonian by a mean-field decoupling. Before moving forward with our results, it is first worthwhile to note that quantum many-body methods such as bosonization and density-matrix renormalization group [101] (DMRG) are used to obtain accurate results in interacting one-dimensional 
systems [102,103]. These methods fully capture quantum fluctuations, which are missing in mean-field approaches. Mean-field theory methods are used to approximately study interacting quasiperiodic models in one dimension, providing qualitatively correct results that can later be refined with more advanced quantum many-body methods. In particular, mean-field methods in one dimension have been benchmarked with density-matrix renormalization group calculations for Aubry-Andre-Harper models [104,105], finding a good qualitative agreement between the mean-field and DMRG methods $[104,105]$. Following those results, it is expected that results obtained with mean-field theory provide a qualitatively correct picture. Specifically, a particular difference between the mean-field and full many-body approaches is that meanfield solutions would predict long-range order, whereas exact solutions would rather show quasi-long-range order, with a power-law decay of correlations in the order parameter [106].

Keeping the previous points in mind, we now move on to solve the previous interacting term given by Eq. (3) using a mean-field approximation $\mathcal{H}_{V}^{\mathrm{MF}}$, including all the normal $\mathcal{H}_{V}^{\mathrm{MF}, h}$ and anomalous $\mathcal{H}_{V}^{\mathrm{MF}, s}, \mathcal{H}_{V}^{\mathrm{MF}, t}$ contributions. The previous mean-field decoupling will give rise to hopping renormalization $\mathcal{H}_{V}^{\mathrm{MF}, h}$, singlet superconductivity renormalization $\mathcal{H}_{V}^{\mathrm{MF}, s}$, and, most importantly, potential spin-triplet superconducting order $\mathcal{H}_{V}^{\mathrm{MF}, t}$. We will focus on this last term, whose contribution to the mean-field Hamiltonian is of the form

$$
\mathcal{H}_{V}^{\mathrm{MF}, t}=\sum_{n} \Delta_{n, n+1}^{s s^{\prime}} c_{n, s} c_{n+1, s^{\prime}}+\text { H.c. },
$$

where, by definition of the fermionic anticommutation relations, $\Delta_{n, n+1}^{s, s^{\prime}}=-\Delta_{n+1, n}^{s^{\prime}, s}$. It is worthwhile to note that the interaction term that we consider can lead to both spin-singlet and spin-triplet components. In our calculations, we have verified that the spin-singlet component is zero in the cases under study. This can be intuitively understood from the fact that the existence of local antiferromagnetism is expected to quench spin-singlet instabilities [34,107-109]. Therefore, whereas spin-singlet instabilities may appear from Eq. (3), the spin-triplet component is the leading instability of the model under study. In the following, we will focus on the spin-triplet component of the superconducting order that fulfills $\Delta_{n, n+1}^{s, s^{\prime}}=$ $-\Delta_{n+1, n}^{s, s^{\prime}}$. As the spin-triplet component of the interactioninduced superconducting state has several degrees of freedom, it is convenient to define a spatially dependent $d$ vector, $\vec{d}_{n, n+1}$, that parameterizes the spin-triplet superconducting order as

$$
\Delta_{n, n+1}^{s s^{\prime}}=\left(\begin{array}{cc}
\Delta_{n, n+1}^{\uparrow \uparrow} & \Delta_{n, n+1}^{\uparrow \downarrow} \\
\Delta_{n, n+1}^{\downarrow \uparrow} & \Delta_{n, n+1}^{\downarrow \downarrow}
\end{array}\right)=i \sigma_{y}\left(\vec{d}_{n, n+1} \cdot \vec{\sigma}\right),
$$

where $\vec{\sigma}$ are the spin-Pauli matrices.

As a first step, it is interesting to look at the real-space distribution of the unconventional superconducting state. In particular, we show in Fig. 3 the real-space distribution of the spin-triplet state defined as

$$
\Xi(n)=\sum_{j}\left|\vec{d}_{n, n+j}\right|^{2}=\left|\vec{d}_{n, n-1}\right|^{2}+\left|\vec{d}_{n, n+1}\right|^{2} .
$$

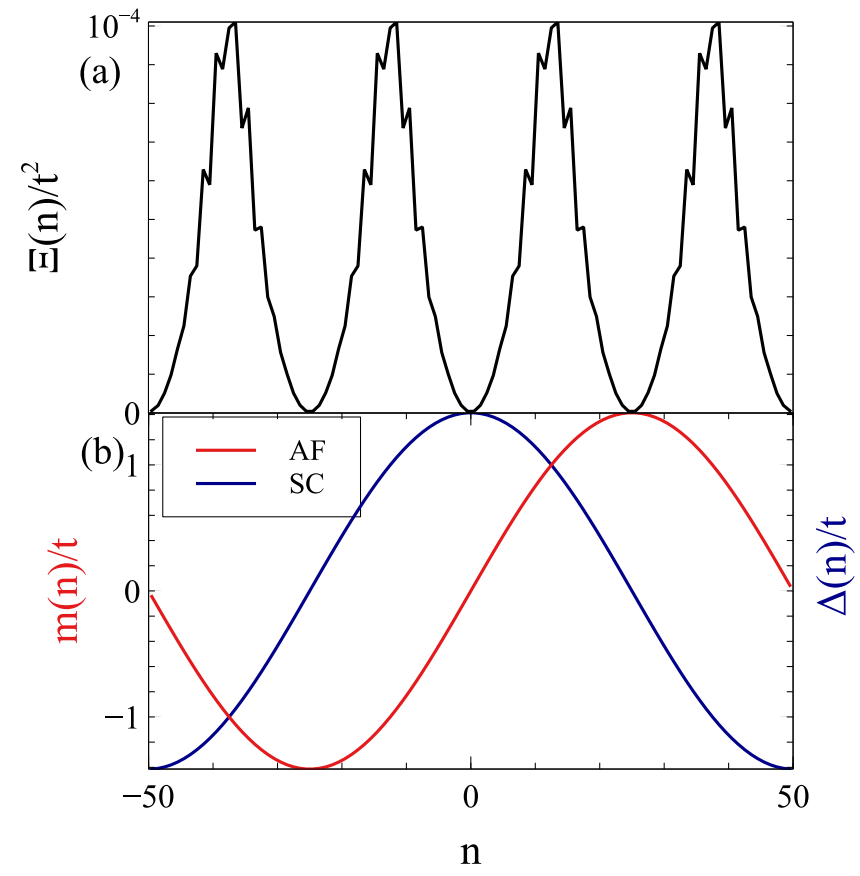

FIG. 3. (a) Self-consistent interaction-induced spin-triplet superconducting order as a function of the position, and (b) spatial profiles of the spin-singlet superconductivity $\Delta(n)=\Delta \cos (\Omega n)$ and antiferromagnetism $m(n)=m \sin (\Omega n)$. It is observed that the spin-triplet component is maximal in the regions where the spin-singlet superconductivity and antiferromagnetism coexist.

We note that in the case of first-neighbor interactions, the previous sum includes only $j=-1$ and $j=+1$, yet in the presence of longer-range interactions, other terms could be included. We observe that the spin-triplet density follows the quasiperiodic pattern, and that it becomes zero in regions only having antiferromagnetism or $s$-wave superconductivity [Figs. 3(a) and 3(b)]. In particular, the value of the spintriplet superconducting order becomes maximal every time the $s$-wave superconductivity and antiferromagnetism coexist maximally in absolute value, irrespective of their signs. Interestingly, we find that such spin-triplet component is maximal right in the region where the $s$-wave superconductivity and antiferromagnetism coexist in the same footing [Figs. 3(a) and 3(b)], highlighting the key interplay of magnetism and superconductivity for driving as a spin-triplet superconducting state. Moreover, it is interesting to examine the specific type of spin-triplet state that the interactions promote. In particular, we find that the $\vec{d}_{n, n+1}$ is always locked to the same direction of the antiferromagnetism, which in terms of the superconducting order parameters is associated to an interaction-induced spin-triplet $\Delta_{n, n+1}^{\uparrow \downarrow}$ order parameter for antiferromagnetism in the $z$ axis.

We now move on to examine the impact of the quasiperiodic criticality on the induced spin-triplet state. As anticipated above, the critical behavior of the wave functions is known to provide an effective mechanism for enhancing electronic instabilities [110-115]. To verify this, we now compute the self-consistent spin-triplet order parameter of Eq. (6) averaged over all the sites, $\langle\Xi\rangle=\frac{1}{N} \sum_{n} \Xi(n)$, as a function of the 

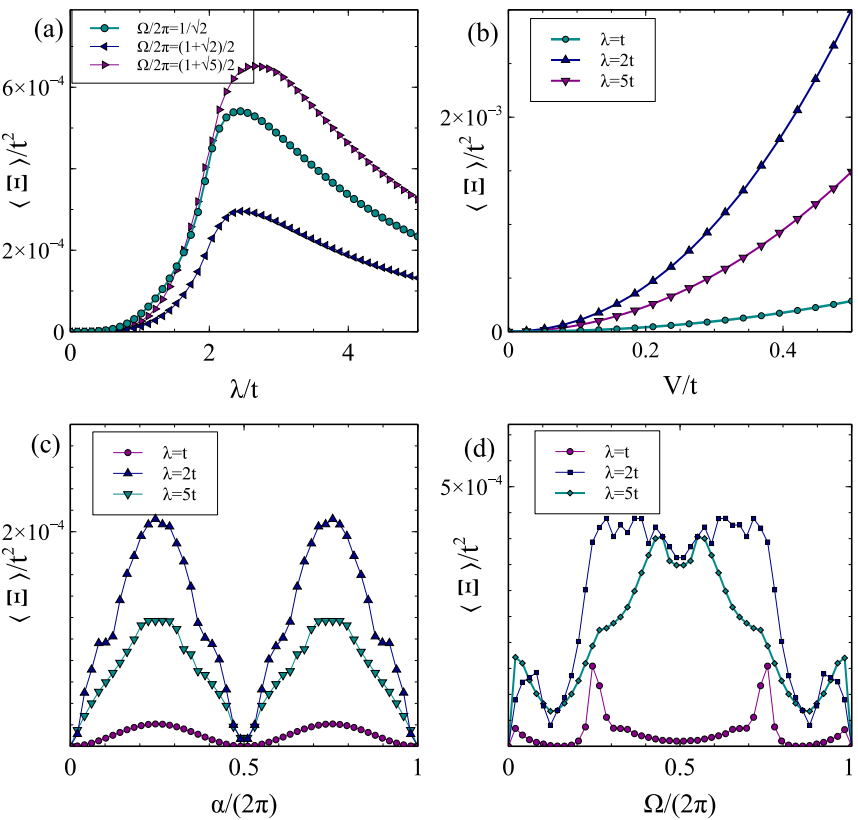

FIG. 4. Self-consistent interaction-induced spin-triplet superconductivity (a) as a function of the superconductor-antiferromagnet (SC-AF) modulation strength for $V=0.2 t, \alpha=\pi / 4$, (b) as a function of the interaction strength $V$ for $\alpha=\pi / 4, \Omega /(2 \pi)=1 / \sqrt{2}$, (c) as a function of the SC-AF angle $\alpha$ for $\Omega /(2 \pi)=1 / \sqrt{2}, V=$ $0.2 t$, and (d) as a function of the quasiperiodic modulation frequency $\Omega$ for $V=0.2 t, \alpha=\pi / 4$. An enhancement of the interactioninduced spin-triplet superconducting state is observed, stemming from the interplay of antiferromagnetism and superconductivity.

modulation strength $\lambda$, as shown in Fig. 4(a). It is observed that as the modulation strength increases, the induced spin-triplet parameter grows, becoming maximal around the critical point and decreasing as the system goes deeper into the localized regime. The enhancement associated to the critical point can also be verified by computing the induced spintriplet order parameter as a function of the interaction strength $V$, as shown in Fig. 4(b). It is seen that for all coupling constants, the spin-triplet state is stronger close to the critical point $\lambda=2 t$ than deep into the extended $(\lambda=t)$ or localized $(\lambda=5 t)$ regime. The fact that the maximum does not exactly appear at the critical point, but towards the localized region, can be rationalized as follows. In the localized limit, a sizable superconducting stability of isolated electronic states can happen at small coupling. In this localized regime, the absence of kinetic energy for the localized modes yields a large expectation value of the superconducting order that explains the existence of a sizable spin-triplet superconductor (SC) in the localized regime, and a maximum that is not exactly at the critical point. However, in this localized regime, the different states that become ordered are spatially separated, which would prevent the existence of phase coherence between them $[106,116]$. While those fluctuations of the phase coherence are not captured at the mean-field level, a calculation that includes quantum fluctuations would show that the phase coherence in this localized regime is very small $[106,116]$. In contrast, for $\lambda<2 t$, the extended nature of the states would give a superconducting state with a large phase coherence and superconducting stiffness.

We now move on to examine the impact of the two quasiperiodic modulations as parameterized by $\alpha$. As we showed above, the extended-localized transition takes place for $\lambda=2 t$, and independently of the value of $\alpha$. This means that a critical point appears independently of the relative strengths between $\Delta$ and $m$, and it only depends on $\lambda=$ $\sqrt{\Delta^{2}+m^{2}}$. In stark contrast, the emergence of a spin-triplet component due to interactions turns out to be highly dependent on $\alpha$, as shown in Fig. 4(c). In particular, we observe that when the system is purely antiferromagnetic or purely superconducting ( $\alpha=0, \pi / 2$ ), the spin-triplet component that is generated is exactly zero. In comparison, the spin-triplet state is maximal for $\alpha=\pi / 4$, which corresponds to having an equal weight on the singlet superconducting and antiferromagnetic order parameters. This observation emphasizes the importance of the coexistence of antiferromagnetism and superconductivity for the emergence of the interaction-induced spin-triplet state.

Finally, we consider the impact of the spatial modulation frequency $\Omega$ in the interaction-induced spin-triplet state. As shown in Fig. 4(d), we observe that the enhancement at the critical point happens for generic values of the modulation frequency. In the limit of small $\Omega$, the system is essentially formed by patches of superconductor and antiferromagnet [87-90], having a typical length of the order of $1 \sim 1 / \Omega$, and thus the interaction between the superconducting and antiferromagnetic state happens in a limited part of the system. In comparison, for $\Omega \approx \pi / 2$, there is a quick oscillation between the two orders, promoting a dense coexistence of antiferromagnetism and spin-singlet superconductivity in the system. We observe that the interaction-induced spin-triplet component is especially strong in this regime [Fig. 4(d)], reflecting the key interplay between spin-singlet superconductivity and antiferromagnetism for driving the unconventional superconducting state.

\section{ROBUSTNESS TO PERTURBATIONS}

In this section, we address the robustness of our phenomenology with respect to perturbations. In particular, we will focus on the impact of next-to-nearest-neighbor hopping and Anderson disorder. The next-to-nearest-neighbor hopping breaks the bipartite nature of the lattice, whereas the Anderson disorder would drive the system to a localized state for all $\lambda$. In particular, we obtain that the critically enhanced spin-triplet superconducting state also happens with those additional perturbations, as elaborated below. Second-neighbor perturbations are expected to appear in a realization of the previous model and break the original bipartite nature of the system. The previous term is included in our Hamiltonian by means of a perturbation of the form

$$
\mathcal{H}_{\mathrm{NNN}}=t^{\prime} \sum_{n, s} c_{n, s}^{\dagger} c_{n+2, s}+\text { H.c. }
$$

The results with this additional perturbation are shown in Fig. 5(a), where we took $t^{\prime}=0.2 t$. It is observed that the enhancement of the interaction-induced spin-triplet state happens in the presence of this additional perturbation. It is 

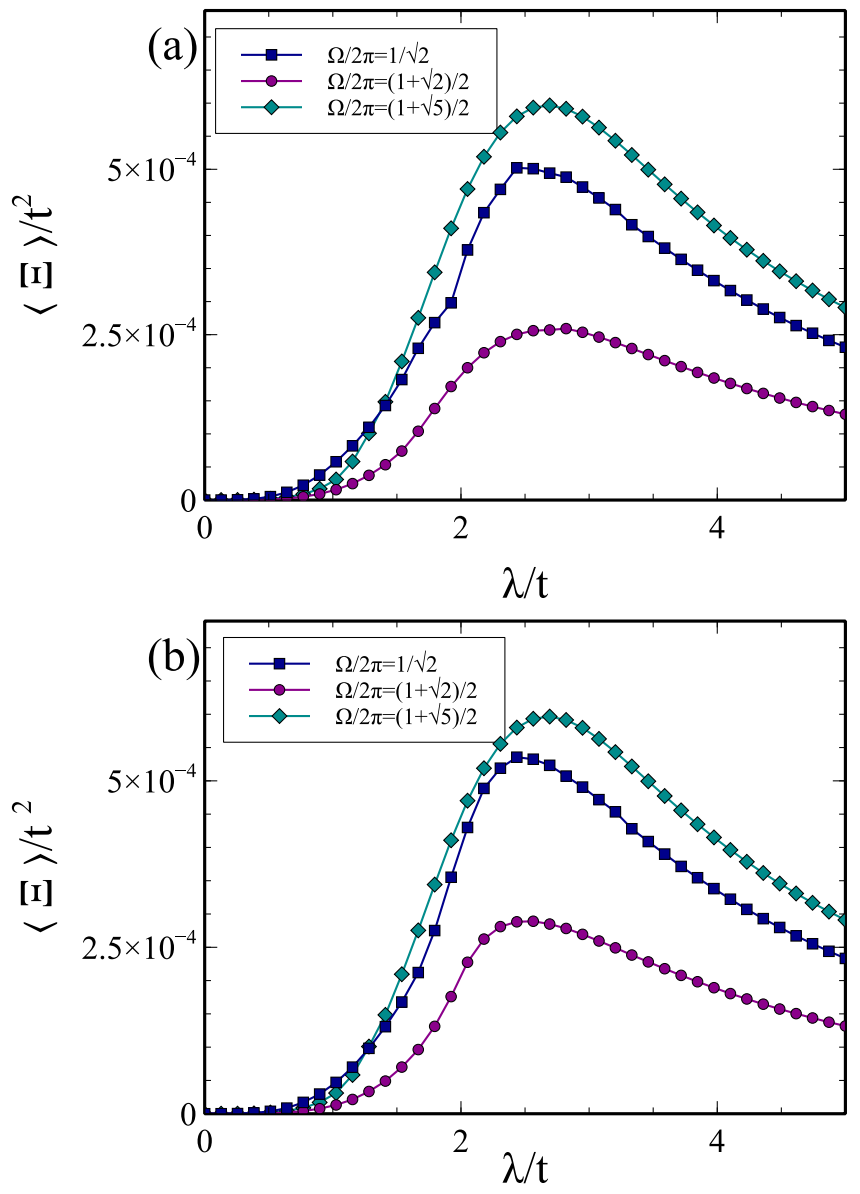

FIG. 5. Spin-triplet superconducting state in the presence of (a) second-neighbor hopping for $\alpha=\pi / 4, V=0.2 t$ and (b) random disorder for $\alpha=\pi / 4, V=0.2 t$. It is observed that the enhancement close to the critical point survives perturbations to the quasiperiodic Hamiltonian of Eq. (1).

worthwhile to note that in the presence of second-neighbor hoppings, the localization-delocalization transition becomes state dependent and will no longer happen at $\lambda=2 t$. Nevertheless, it is observed that the qualitative behavior remains analogous to the idealized case with $t^{\prime}=0$. This is especially important for potential realizations of our model in twisted two-dimensional materials and cold-atom setups, as generically these systems present small additional contributions to the Hamiltonian such as a second-neighbor hopping.

Next, we consider the impact of random disorder in the system, included as an on-site Anderson perturbation,

$$
\mathcal{H}_{\mathrm{dis}}=\sum_{n, s} \epsilon_{n} c_{n, s}^{\dagger} c_{n, s},
$$

where $\epsilon_{n}$ is a random number between $[-0.1,0.1] t$. First, it is interesting to note that the inclusion of an arbitrarily small amount of disorder would drive the extended states to a localized regime. As a result, in the presence of disorder, the localization-delocalization transition as a function of $\lambda$ is completely destroyed, as the state becomes localized for all $\lambda$. The disorder strength $\lambda$ will define a minimal localization length for the system. As $\lambda$ is ramped up, the system will go from a localized regime dominated by the disorder to a regime in which the localization is dominated by the quasiperiodic potential. Although the critical point is washed out, the enhancement of the superconducting state will still be visible at this quasiperiodic-disorder localization crossover. This is shown in Fig. 5(b), where it is seen that the spin-triplet enhancement close to the former critical point is still visible. This phenomenology shows that even in experimental setups that host small imperfections, the enhancement of an unconventional spin-triplet superconducting state can be observed.

Finally, we comment on the prospects of extending the previous phenomenology to two-dimensional systems. In our manuscript, we focused on showing the existence of a localization-delocalization transition for a quasiperiodic onedimensional model. Right at the transition, the existence of a critical point leads to an enhancement of an interactioninduced instability that, due to the nature of our system, was a spin-triplet superconducting instability. Interestingly, a quasiperiodic system in higher dimensions also shows localization-delocalization transitions, and associated critical points to them [117]. Enhanced symmetry-broken orders induced by interactions have been found in those quasiperiodic two-dimensional models [38,118-122], both in the cases of spin-singlet superconductivity [38,118-120] and magnetic order [122]. The previous phenomenology in two dimensions was also demonstrated for one-dimensional models, highlighting that symmetry breaking enhanced by quasiperiodicity happens both in one-dimensional and two-dimensional quasiperiodic models. Ultimately, the previous results suggest that the extension of our model to two dimensions could lead to two-dimensional spin-triplet superconductivity.

\section{CONCLUSION}

To summarize, we have demonstrated that antiferromagnet-superconductor moiré patterns show a critical point associated with a localization-delocalization transition. We showed that the quasiperiodic criticality happens for arbitrary ratios between the superconducting and antiferromagnetic order parameters, and that the critical point is universally located in a curve defined by the two order parameters. Upon inclusion of residual electronic interactions, we demonstrated the emergence of an unconventional spin-triplet state, whose $d$ vector is locked along the antiferromagnetic spin direction. We showed that the emergence of this unconventional superconducting state is finely related to the interplay between antiferromagnetism and superconductivity, having a spatially inhomogeneous superconducting order that is maximal when the two parent orders coexist. Finally, we showed that this phenomenology happens for generic quasiperiodic modulation frequencies and survives the presence of perturbations to the Hamiltonian. Ultimately, the phenomenology that is presented can be realized in twisted graphene superlattices with atomically engineered impurities, and generically on moiré patterns between two-dimensional antiferromagnets and superconductors. Our results put forward antiferromagnetic-superconducting quasiperiodicity as a powerful knob to engineer robust superconducting states, providing a route towards the design of artificial unconventional superconductors. 


\section{ACKNOWLEDGMENTS}

We acknowledge the computational resources provided by the Aalto Science-IT project. We thank P. Liljeroth,
O. Zilberberg, A. Strkalj, M. Sigrist, and A. Ramires for useful discussions. J.L.L. is grateful for financial support from the Academy of Finland Projects No. 331342 and No. 336243.
[1] M. Sigrist and K. Ueda, Phenomenological theory of unconventional superconductivity, Rev. Mod. Phys. 63, 239 (1991).

[2] J. Linder, Y. Tanaka, T. Yokoyama, A. Sudbø, and N. Nagaosa, Unconventional Superconductivity On A Topological Insulator, Phys. Rev. Lett. 104, 067001 (2010).

[3] D. Aasen, M. Hell, R. V. Mishmash, A. Higginbotham, J. Danon, M. Leijnse, T. S. Jespersen, J. A. Folk, C. M. Marcus, K. Flensberg, and J. Alicea, Milestones Toward MajoranaBased Quantum Computing, Phys. Rev. X 6, 031016 (2016).

[4] J. Alicea, Y. Oreg, G. Refael, F. von Oppen, and M. P. A. Fisher, Non-Abelian statistics and topological quantum information processing in 1D wire networks, Nat. Phys. 7, 412 (2011).

[5] Ch. Wälti, H. R. Ott, Z. Fisk, and J. L. Smith, Spectroscopic Evidence For Unconventional Superconductivity In $\mathrm{UBe}_{13}$, Phys. Rev. Lett. 84, 5616 (2000).

[6] G.-Q. Zheng, N. Yamaguchi, H. Kan, Y. Kitaoka, J. L. Sarrao, P. G. Pagliuso, N. O. Moreno, and J. D. Thompson, Coexistence of antiferromagnetic order and unconventional superconductivity in heavy-fermion $\mathrm{CeRh}_{1-x} \mathrm{Ir}_{x} \mathrm{In}_{5}$ compounds: Nuclear quadrupole resonance studies, Phys. Rev. B 70, 014511 (2004)

[7] J. F. Landaeta, D. Subero, D. Catalá, S. V. Taylor, N. Kimura, R. Settai, Y. Ōnuki, M. Sigrist, and I. Bonalde, Unconventional superconductivity and quantum criticality in the heavy fermions $\mathrm{CeIrSi}_{3}$ and $\mathrm{CeRhSi}_{3}$, Phys. Rev. B 97, 104513 (2018).

[8] S. Kawasaki, M. Yashima, Y. Kitaoka, K. Takeda, K. Shimizu, Y. Oishi, M. Takata, T. C. Kobayashi, H. Harima, S. Araki, H. Shishido, R. Settai, and Y. Ōnuki, Pressure-induced unconventional superconductivity in the heavy-fermion antiferromagnet $\mathrm{CeIn}_{3}$ : An ${ }^{115}$ In-NQR study under pressure, Phys. Rev. B 77, 064508 (2008).

[9] T. Yoshida, A. Daido, Y. Yanase, and N. Kawakami, Fate Of

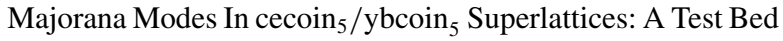
For The Reduction Of Topological Classification, Phys. Rev. Lett. 118, 147001 (2017).

[10] K. Matano, M. Kriener, K. Segawa, Y. Ando, and G. qing Zheng, Spin-rotation symmetry breaking in the superconducting state of $\mathrm{Cu}_{x} \mathrm{Bi}_{2} \mathrm{Se}_{3}$, Nat. Phys. 12, 852 (2016).

[11] S. Yonezawa, K. Tajiri, S. Nakata, Y. Nagai, Z. Wang, K. Segawa, Y. Ando, and Y. Maeno, Thermodynamic evidence for nematic superconductivity in $\mathrm{Cu}_{x} \mathrm{Bi}_{2} \mathrm{Se}_{3}$, Nat. Phys. 13, 123 (2016).

[12] L. Fu, Odd-parity topological superconductor with nematic order: Application to $\mathrm{Cu}_{x} \mathrm{Bi}_{2} \mathrm{Se}_{3}$, Phys. Rev. B 90, 100509(R) (2014).

[13] J. W. F. Venderbos, V. Kozii, and L. Fu, Odd-parity superconductors with two-component order parameters: Nematic and chiral, full gap, and Majorana node, Phys. Rev. B 94, 180504(R) (2016)

[14] L. Jiao, S. Howard, S. Ran, Z. Wang, J. O. Rodriguez, M. Sigrist, Z. Wang, N. P. Butch, and V. Madhavan, Chiral superconductivity in heavy-fermion metal $\mathrm{UTe}_{2}$, Nature (London) 579, 523 (2020).

[15] K. E. Avers, W. J. Gannon, S. J. Kuhn, W. P. Halperin, J. A. Sauls, L. DeBeer-Schmitt, C. D. Dewhurst, J. Gavilano, G. Nagy, U. Gasser, and M. R. Eskildsen, Broken time-reversal symmetry in the topological superconductor $\mathrm{UPt}_{3}$, Nat. Phys. 16, 531 (2020).

[16] S. Ran, H. Kim, I.-L. Liu, S. R. Saha, I. Hayes, T. Metz, Y. S. Eo, J. Paglione, and N. P. Butch, Enhancement and reentrance of spin triplet superconductivity in $\mathrm{UTe}_{2}$ under pressure, Phys. Rev. B 101, 140503(R) (2020).

[17] F. Laube, G. Goll, H. v. Löhneysen, M. Fogelström, and F. Lichtenberg, Spin-Triplet Superconductivity In $\mathrm{SR}_{2} \mathrm{RuO}_{4}$ Probed By Andreev Reflection, Phys. Rev. Lett. 84, 1595 (2000).

[18] W. Higemoto, S. R. Saha, A. Koda, K. Ohishi, R. Kadono, Y. Aoki, H. Sugawara, and H. Sato, Spin-triplet superconductivity in $\mathrm{PrOs}_{4} \mathrm{Sb}_{12}$ probed by muon knight shift, Phys. Rev. B 75, 020510(R) (2007).

[19] M. Manago, S. Kitagawa, K. Ishida, K. Deguchi, N. K. Sato, and T. Yamamura, Spin-triplet superconductivity in the paramagnetic ucoge under pressure studied by ${ }^{59}$ Co NMR, Phys. Rev. B 100, 035203 (2019).

[20] H. Tou, Y. Kitaoka, K. Ishida, K. Asayama, N. Kimura, Y. Onuki, E. Yamamoto, Y. Haga, and K. Maezawa, Nonunitary Spin-Triplet Superconductivity In $\mathrm{UPt}_{3}$ : Evidence From ${ }^{195} \mathrm{Pt}$ Knight Shift Study, Phys. Rev. Lett. 80, 3129 (1998).

[21] K. Ishida, D. Ozaki, T. Kamatsuka, H. Tou, M. Kyogaku, Y. Kitaoka, N. Tateiwa, N. K. Sato, N. Aso, C. Geibel, and F. Steglich, Spin-Triplet Superconductivity In $\mathrm{UNi}_{2} \mathrm{Al}_{3}$ Revealed By The ${ }^{27} A l$ Knight Shift Measurement, Phys. Rev. Lett. 89, 037002 (2002).

[22] J. Alicea, New directions in the pursuit of Majorana fermions in solid state systems, Rep. Prog. Phys. 75, 076501 (2012).

[23] C. W. J. Beenakker, Search for Majorana fermions in superconductors, Annu. Rev. Condens. Matter Phys. 4, 113 (2013).

[24] X.-L. Qi and S.-C. Zhang, Topological insulators and superconductors, Rev. Mod. Phys. 83, 1057 (2011).

[25] R. M. Lutchyn, J. D. Sau, and S. Das Sarma, Majorana Fermions And A Topological Phase Transition In Semiconductor-Superconductor Heterostructures, Phys. Rev. Lett. 105, 077001 (2010).

[26] Y. Oreg, G. Refael, and F. von Oppen, Helical Liquids And Majorana Bound States In Quantum Wires, Phys. Rev. Lett. 105, 177002 (2010).

[27] J. D. Sau, R. M. Lutchyn, S. Tewari, and S. Das Sarma, Generic New Platform For Topological Quantum Computation Using Semiconductor Heterostructures, Phys. Rev. Lett. 104, 040502 (2010).

[28] L. Fu and C. L. Kane, Superconducting Proximity Effect And Majorana Fermions At The Surface Of A Topological Insulator, Phys. Rev. Lett. 100, 096407 (2008). 
[29] B. E. Feldman, M. T. Randeria, J. Li, S. Jeon, Y. Xie, Z. Wang, I. K. Drozdov, B. A. Bernevig, and A. Yazdani, Highresolution studies of the Majorana atomic chain platform, Nat. Phys. 13, 286 (2016).

[30] H. Zhang, C.-X. Liu, S. Gazibegovic, Di. Xu, J. A. Logan, G. Wang, N. van Loo, J. D. S. Bommer, M. W. A. de Moor, D. Car, R. L. M. Op. het Veld, P. J. van Veldhoven, S. Koelling, M. A. Verheijen, M. Pendharkar, D. J. Pennachio, B. Shojaei, J. S. Lee, C. J. Palmstrøm, E. P. A. M. Bakkers, S. D. Sarma, and L. P. Kouwenhoven, Quantized Majorana conductance, Nature (London) 556, 74 (2018).

[31] S. Kezilebieke, Md. Nurul Huda, V. Vaňo, M. Aapro, S. C. Ganguli, O. J. Silveira, S. Głodzik, A. S. Foster, T. Ojanen, and P. Liljeroth, Topological superconductivity in a van der Waals heterostructure, Nature 588, 424 (2020).

[32] I. I. Satija and G. G. Naumis, Chern and Majorana modes of quasiperiodic systems, Phys. Rev. B 88, 054204 (2013).

[33] W. DeGottardi, D. Sen, and S. Vishveshwara, Majorana Fermions In Superconducting 1d Systems Having Periodic, Quasiperiodic, And Disordered Potentials, Phys. Rev. Lett. 110, 146404 (2013).

[34] P. W. Anderson, Theory of dirty superconductors, J. Phys. Chem. Solids 11, 26 (1959).

[35] Y. J. Rosen, A. Sharoni, and I. K. Schuller, Enhanced superconducting vortex pinning with disordered nanomagnetic arrays, Phys. Rev. B 82, 014509 (2010).

[36] Y. L. Wang, L. R. Thoutam, Z. L. Xiao, B. Shen, J. E. Pearson, R. Divan, L. E. Ocola, G. W. Crabtree, and W. K. Kwok, Enhancing superconducting critical current by randomness, Phys. Rev. B 93, 045111 (2016).

[37] M. G. Karkut, J. M. Triscone, D. Ariosa, and Ø. Fischer, Quasiperiodic metallic multilayers: Growth and superconductivity, Phys. Rev. B 34, 4390 (1986).

[38] S. Sakai, N. Takemori, A. Koga, and R. Arita, Superconductivity on a quasiperiodic lattice: Extended-to-localized crossover of cooper pairs, Phys. Rev. B 95, 024509 (2017).

[39] K. M. Wong, E. Lopdrup, J. L. Wagner, Y. Shen, and S. J. Poon, Transport and superconducting properties of the $\mathrm{Mg}_{32}(\mathrm{Al}, \mathrm{Zn})_{49}$-type quasicrystalline and crystalline phases, Phys. Rev. B 35, 2494 (1987).

[40] J. L. Wagner, B. D. Biggs, K. M. Wong, and S. J. Poon, Specific-heat and transport properties of alloys exhibiting quasicrystalline and crystalline order, Phys. Rev. B 38, 7436 (1988).

[41] D. Shechtman, I. Blech, D. Gratias, and J. W. Cahn, Metallic Phase With Long-Range Orientational Order And No Translational Symmetry, Phys. Rev. Lett. 53, 1951 (1984).

[42] D. Levine and P. J. Steinhardt, Quasicrystals: A New Class Of Ordered Structures, Phys. Rev. Lett. 53, 2477 (1984).

[43] A. Jagannathan and F. Piéchon, Energy levels and their correlations in quasicrystals, Philos. Mag. 87, 2389 (2007).

[44] M. Kohmoto and B. Sutherland, Electronic States On A Penrose Lattice, Phys. Rev. Lett. 56, 2740 (1986).

[45] M. Arai, T. Tokihiro, T. Fujiwara, and M. Kohmoto, Strictly localized states on a two-dimensional penrose lattice, Phys. Rev. B 38, 1621 (1988).

[46] T. Rieth and M. Schreiber, Identification of spatially confined states in two-dimensional quasiperiodic lattices, Phys. Rev. B 51, 15827 (1995).
[47] B. Sutherland, Self-similar ground-state wave function for electrons on a two-dimensional penrose lattice, Phys. Rev. B 34, 3904 (1986).

[48] H. Tsunetsugu, T. Fujiwara, K. Ueda, and T. Tokihiro, Eigenstates in 2-dimensional Penrose tiling, J. Phys. Soc. Jpn. 55, 1420 (1986)

[49] T. Tokihiro, T. Fujiwara, and M. Arai, Exact eigenstates on a two-dimensional Penrose lattice and their fractal dimensions, Phys. Rev. B 38, 5981 (1988).

[50] T. Fujiwara, Electronic structure in the Al-Mn alloy crystalline analog of quasicrystals, Phys. Rev. B 40, 942 (1989).

[51] T. Fujiwara and T. Yokokawa, Universal Pseudogap At Fermi Energy In Quasicrystals, Phys. Rev. Lett. 66, 333 (1991).

[52] A. Ishikawa, Y. Takagiwa, K. Kimura, and R. Tamura, Probing of the pseudogap via thermoelectric properties in the Au-AlGd quasicrystal approximant, Phys. Rev. B 95, 104201 (2017).

[53] S. Jazbec, S. Vrtnik, Z. Jagliāić, S. Kashimoto, J. Ivkov, P. Popāević, A. Smontara, H. J. Kim, J. G. Kim, and J. Dolinšek, Electronic density of states and metastability of icosahedral Au-Al-Yb quasicrystal, J. Alloys Compd. 586, 343 (2014).

[54] F. S. Pierce, S. J. Poon, and Q. Guo, Electron localization in metallic quasicrystals, Science 261, 737 (1993).

[55] G. Trambly de Laissardière and T. Fujiwara, Electronic structure and transport in a model approximant of the decagonal quasicrystal Al-Cu-Co, Phys. Rev. B 50, 9843 (1994).

[56] T. Timusk, J. P. Carbotte, C. C. Homes, D. N. Basov, and S. G. Sharapov, Three-dimensional Dirac fermions in quasicrystals as seen via optical conductivity, Phys. Rev. B 87, 235121 (2013).

[57] G. T. de Laissardière and D. Mayou, Anomalous electronic transport in quasicrystals and related complex metallic alloys, C. R. Phys. 15, 70 (2014).

[58] A. P. Siebesma and L. Pietronero, Multifractal properties of wave functions for one-dimensional systems with an incommensurate potential, Europhys. Lett. 4, 597 (1987).

[59] S. Aubry and G. André, Analyticity breaking and Anderson localization in incommensurate lattices, Ann. Israel Phys. Soc 3, 18 (1980)

[60] T. Devakul and D. A. Huse, Anderson localization transitions with and without random potentials, Phys. Rev. B 96, 214201 (2017).

[61] Y. Su and S.-Z. Lin, Nontrivial topology and localization in the double exchange model with possible applications to perovskite manganites, Phys. Rev. B 98, 235116 (2018).

[62] Y. E. Kraus, Y. Lahini, Z. Ringel, M. Verbin, and O. Zilberberg, Topological States And Adiabatic Pumping In Quasicrystals, Phys. Rev. Lett. 109, 106402 (2012).

[63] Y. E. Kraus and O. Zilberberg, Topological Equivalence Between The Fibonacci Quasicrystal And The Harper Model, Phys. Rev. Lett. 109, 116404 (2012).

[64] Y. Cao, V. Fatemi, S. Fang, K. Watanabe, T. Taniguchi, E. Kaxiras, and P. Jarillo-Herrero, Unconventional superconductivity in magic-angle graphene superlattices, Nature (London) 556, 43 (2018).

[65] M. Yankowitz, S. Chen, H. Polshyn, Y. Zhang, K. Watanabe, T. Taniguchi, D. Graf, A. F. Young, and C. R. Dean, Tuning superconductivity in twisted bilayer graphene, Science 363, 1059 (2019).

[66] X. Lu, P. Stepanov, W. Yang, M. Xie, M. A. Aamir, I. Das, C. Urgell, K. Watanabe, T. Taniguchi, G. Zhang, A. Bachtold, 
A. H. MacDonald, and D. K. Efetov, Superconductors, orbital magnets and correlated states in magic-angle bilayer graphene, Nature (London) 574, 653 (2019).

[67] B. Roy and V. Juričić, Unconventional superconductivity in nearly flat bands in twisted bilayer graphene, Phys. Rev. B 99, 121407(R) (2019).

[68] C. Xu and L. Balents, Topological Superconductivity In Twisted Multilayer Graphene, Phys. Rev. Lett. 121, 087001 (2018).

[69] A. Julku, T. J. Peltonen, L. Liang, T. T. Heikkilä, and P. Törmä, Superfluid weight and Berezinskii-Kosterlitz-Thouless transition temperature of twisted bilayer graphene, Phys. Rev. B 101, 060505(R) (2020).

[70] T. J. Peltonen, R. Ojajärvi, and T. T. Heikkilä, Mean-field theory for superconductivity in twisted bilayer graphene, Phys. Rev. B 98, 220504(R) (2018).

[71] H. González-Herrero, J. Gómez-Rodríguez, P. Mallet, M. Moaied, J. J. Palacios, C. Salgado, M. M. Ugeda, J.-Y. Veuillen, F. Yndurain, and I. Brihuega, Atomic-scale control of graphene magnetism by using hydrogen atoms, Science 352, 437 (2016).

[72] R. Toskovic, R. van den Berg, A. Spinelli, I. S. Eliens, B. van den Toorn, B. Bryant, J.-S. Caux, and A. F. Otte, Atomic spinchain realization of a model for quantum criticality, Nat. Phys. 12, 656 (2016).

[73] S. Kezilebieke, R. Žitko, M. Dvorak, T. Ojanen, and P. Liljeroth, Observation of coexistence of Yu-Shiba-Rusinov states and spin-flip excitations, Nano Lett. 19, 4614 (2019).

[74] S. Loth, S. Baumann, C. P. Lutz, D. M. Eigler, and A. J. Heinrich, Bistability in atomic-scale antiferromagnets, Science 335, 196 (2012).

[75] E. Liebhaber, S. A. González, R. Baba, G. Reecht, B. W. Heinrich, S. Rohlf, K. Rossnagel, F. von Oppen, and K. J. Franke, Yu-Shiba-Rusinov states in the charge-density modulated superconductor $\mathrm{NbSe}_{2}$, Nano Lett. 20, 339 (2019).

[76] M. Ruby, F. Pientka, Y. Peng, F. von Oppen, B. W. Heinrich, and K. J. Franke, End States And Subgap Structure In Proximity-Coupled Chains Of Magnetic Adatoms, Phys. Rev. Lett. 115, 197204 (2015).

[77] E. C. del Río, P. Mallet, H. González-Herrero, J. L. Lado, J. Fernández-Rossier, J. M. Gómez-Rodríguez, J.-Y. Veuillen, and I. Brihuega, Quantum confinement of Dirac quasiparticles in graphene patterned with sub-nanometer precision, Adv. Mater. 32, 2001119 (2020).

[78] D.-J. Choi, N. Lorente, J. Wiebe, K. von Bergmann, A. F. Otte, and A. J. Heinrich, Colloquium: Atomic spin chains on surfaces, Rev. Mod. Phys. 91, 041001 (2019).

[79] E. Kogan, RKKY interaction in graphene, Phys. Rev. B 84, 115119 (2011).

[80] O. V. Yazyev and A. Pasquarello, Metal adatoms on graphene and hexagonal boron nitride: Towards rational design of selfassembly templates, Phys. Rev. B 82, 045407 (2010).

[81] N. A. García-Martínez and J. Fernández-Rossier, Designer fermion models in functionalized graphene bilayers, Phys. Rev. Research 1, 033173 (2019).

[82] J. L. Lado and O. Zilberberg, Topological spin excitations in Harper-Heisenberg spin chains, Phys. Rev. Research 1, 033009 (2019).

[83] I. Brihuega and F. Yndurain, Selective hydrogen adsorption in graphene rotated bilayers, J. Phys. Chem. B 122, 595 (2017).
[84] A. Lopez-Bezanilla and J. L. Lado, Defect-induced magnetism and Yu-Shiba-Rusinov states in twisted bilayer graphene, Phys. Rev. Materials 3, 084003 (2019).

[85] A. Ramires and J. L. Lado, Impurity-induced triple point fermions in twisted bilayer graphene, Phys. Rev. B 99, 245118 (2019).

[86] A. Lopez-Bezanilla and J. L. Lado, Electrical band flattening, valley flux, and superconductivity in twisted trilayer graphene, Phys. Rev. Research 2, 033357 (2020).

[87] J. L. Lado and M. Sigrist, Solitonic in-gap modes in a superconductor-quantum antiferromagnet interface, Phys. Rev. Research 2, 023347 (2020).

[88] J. L. Lado and M. Sigrist, Two-Dimensional Topological Superconductivity With Antiferromagnetic Insulators, Phys. Rev. Lett. 121, 037002 (2018).

[89] A. L. R. Manesco, G. Weber, and D. Rodrigues, Effective model for Majorana modes in graphene, Phys. Rev. B 100, 125411 (2019).

[90] P. San-Jose, J. L. Lado, R. Aguado, F. Guinea, and J. Fernández-Rossier, Majorana Zero Modes In Graphene, Phys. Rev. X 5, 041042 (2015).

[91] P. W. Anderson, Absence of diffusion in certain random lattices, Phys. Rev. 109, 1492 (1958).

[92] M. Kohmoto, L. P. Kadanoff, and C. Tang, Localization Problem In One Dimension: Mapping And Escape, Phys. Rev. Lett. 50, 1870 (1983).

[93] S. S. Luntama, P. Törmä, and J. L. Lado, Interactioninduced topological superconductivity in antiferromagnetsuperconductor junctions, Phys. Rev. Research 3, 012021 (2021).

[94] Lu. Zhou, H. Pu, and W. Zhang, Anderson localization of cold atomic gases with effective spin-orbit interaction in a quasiperiodic optical lattice, Phys. Rev. A 87, 023625 (2013).

[95] V. Goblot, A. Štrkalj, N. Pernet, J. L. Lado, C. Dorow, A. Lemaître, L. Le Gratiet, A. Harouri, I. Sagnes, S. Ravets, A. Amo, J. Bloch, and O. Zilberberg, Emergence of criticality through a cascade of delocalization transitions in quasiperiodic chains, Nat. Phys. 16, 832 (2020).

[96] M. Johansson and R. Riklund, Mobility edges and multifractal properties in a one-dimensional system with three incommensurate frequencies, Phys. Rev. B 42, 8244 (1990).

[97] J. Wang, X.-Ji. Liu, G. Xianlong, and H. Hu, Phase diagram of a non-Abelian Aubry-André-Harper model with $p$-wave superfluidity, Phys. Rev. B 93, 104504 (2016).

[98] H. Q. Yuan, U. Grimm, P. Repetowicz, and M. Schreiber, Energy spectra, wave functions, and quantum diffusion for quasiperiodic systems, Phys. Rev. B 62, 15569 (2000)

[99] C. Castellani and L. Peliti, Multifractal wavefunction at the localisation threshold, J. Phys. A: Math. Gen. 19, L429 (1986).

[100] V. I Fal'ko and K. B Efetov, Multifractality: Generic property of eigenstates of 2D disordered metals, Europhys. Lett. 32, 627 (1995).

[101] S. R. White, Density Matrix Formulation For Quantum Renormalization Groups, Phys. Rev. Lett. 69, 2863 (1992).

[102] J. Vidal, D. Mouhanna, and T. Giamarchi, Correlated Fermions In A One-Dimensional Quasiperiodic Potential, Phys. Rev. Lett. 83, 3908 (1999). 
[103] C. Schuster, R. A. Römer, and M. Schreiber, Interacting particles at a metal-insulator transition, Phys. Rev. B 65, 115114 (2002).

[104] Y. E. Kraus, O. Zilberberg, and R. Berkovits, Enhanced compressibility due to repulsive interaction in the harper model, Phys. Rev. B 89, 161106(R) (2014).

[105] B.-el. Friedman and R. Berkovits, Compressibility enhancement in an almost staggered interacting harper model, Phys. Rev. B 91, 104203 (2015).

[106] M. Tezuka and A. M. García-García, Stability of the superfluid state in a disordered one-dimensional ultracold fermionic gas, Phys. Rev. A 82, 043613 (2010).

[107] P. W. Anderson, Structure of "triplet" superconducting energy gaps, Phys. Rev. B 30, 4000 (1984).

[108] A. Ramires, D. F. Agterberg, and M. Sigrist, Tailoring $T_{c}$ by symmetry principles: The concept of superconducting fitness, Phys. Rev. B 98, 024501 (2018).

[109] L. Andersen, A. Ramires, Z. Wang, T. Lorenz, and Y. Ando, Generalized Anderson's theorem for superconductors derived from topological insulators, Sci. Adv. 6, eaay6502 (2020).

[110] N. Takemori, R. Arita, and S. Sakai, Physical properties of weak-coupling quasiperiodic superconductors, Phys. Rev. B 102, 115108 (2020).

[111] C. Rubio-Verdú, A. M. Garcia-Garcia, H. Ryu, D.-J. Choi, J. Zaldivar, S. Tang, Bo. Fan, Z.-X. Shen, S.-K. Mo, J. I. Pascual, and M. M. Ugeda, Visualization of multifractal superconductivity in a two-dimensional transition metal dichalcogenide in the weak-disorder regime, Nano Lett. 20, 5111 (2020).

[112] L. Dell'Anna, Enhancement of critical temperatures in disordered bipartite lattices, Phys. Rev. B 88, 195139 (2013).

[113] I. S. Burmistrov, I. V. Gornyi, and A. D. Mirlin, Enhancement Of The Critical Temperature Of Superconductors
By Anderson Localization, Phys. Rev. Lett. 108, 017002 (2012).

[114] A. P. Petrović, D. Ansermet, D. Chernyshov, M. Hoesch, D. Salloum, P. Gougeon, M. Potel, L. Boeri, and C. Panagopoulos, A disorder-enhanced quasi-one-dimensional superconductor, Nat. Commun. 7, 12262 (2016).

[115] Bo. Fan and A. M. García-García, Superconductivity at the three-dimensional Anderson metal-insulator transition, Phys. Rev. B 102, 184507 (2020).

[116] Bo. Fan and A. M. García-García, Enhanced phase-coherent multifractal two-dimensional superconductivity, Phys. Rev. B 101, 104509 (2020).

[117] N. Macé, A. Jagannathan, P. Kalugin, R. Mosseri, and F. Piéchon, Critical eigenstates and their properties in oneand two-dimensional quasicrystals, Phys. Rev. B 96, 045138 (2017).

[118] R. N. Araújo and E. C. Andrade, Conventional superconductivity in quasicrystals, Phys. Rev. B 100, 014510 (2019).

[119] S. Sakai and R. Arita, Exotic pairing state in quasicrystalline superconductors under a magnetic field, Phys. Rev. Research 1, 022002(R) (2019).

[120] Ye. Cao, Y. Zhang, Yu.-Bo. Liu, C.-C. Liu, W.-Q. Chen, and F. Yang, Kohn-Luttinger Mechanism Driven Exotic Topological Superconductivity On The Penrose Lattice, Phys. Rev. Lett. 125, 017002 (2020).

[121] R. Ghadimi, T. Sugimoto, and T. Tohyama, Mean-field study of the Bose-Hubbard model in the Penrose lattice, Phys. Rev. B 102, 224201 (2020).

[122] A. Koga, Superlattice structure in the antiferromagnetically ordered state in the Hubbard model on the Ammann-Beenker tiling, Phys. Rev. B 102, 115125 (2020). 